\title{
EL PROBLEMA DE LAS CLASES NATURALES EN QUÍMICA: ALGUNAS DIFICULTADES PARA EL MICROESTRUCTURALISMO
}

\author{
Juan Camilo Martínez González \\ CONICET \\ olimac62@hotmail.com \\ MARIANA Córdoba \\ Universidad de Buenos Aires \\ CONICET \\ mariana.cordoba.revah@gmail.com
}

RESUMEN: Nuestro propósito es trastornar el tratamiento habitual del problema de las clases naturales en filosofía de la ciencia, analizándolo sincrónicamente y poniendo la química en el foco. Analizaremos tres problemas que la disciplina presenta al microesencialismo: (1) la insuficiencia del número atómico como propiedad y criterio esencial, (2) la imposibilidad de la mecánica cuántica de dar cuenta de la estructura molecular y, por consiguiente, de importantes propiedades macroscópicas de las sustancias, (3) la imposibilidad de definir propiedades centrales, como la acidez, a partir de las propiedades microscópicas. Argumentaremos que, sin embargo, cabe mantener la noción de clase natural en química, pero reformulándola desde la perspectiva del realismo activo y el pluralismo ideológico de Hasok Chang. Esta propuesta permite pensar que si bien las clasificaciones no deben ser interpretadas en un sentido esencialista, no son arbitrarias ni meramente convencionales, porque la realidad puede hacer fracasar un sistema de clasificación y dar éxito a otro.

PALABRAS CLAVE: microesencialismo, número atómico, estructura molecular, ácidos, pluralismo

SUMMARY: Our purpose is to subvert the traditional analysis of the problem of natural kinds in philosophy of science, which will be analyzed from a synchronic perspective and focusing on chemistry. Three problems of essentialist microstructuralism in chemistry will be considered: (1) the atomic number is insufficient as an essential property and criterion, (2) quantum mechanics cannot account for molecular shape and, hence, for important macroscopic properties of substances, (3) it is not possible to define relevant properties, as acidity, from microscopic ones. We will argue it is convenient to maintain the notion of natural kind, though, but it can be redefined from the perspective of active realism and ideological pluralism proposed by Hasok Chang. This approach allows us to think that classifications must not be considered from an essentialist approach, but they are not arbitrary or merely conventional either, since reality can make a classification system fail and make another one work.

KEY WORDS: microstructuralism, atomic number, molecular shape, acids, pluralism 


\section{Introducción}

El problema de la existencia de clases naturales y la cuestión de su persistencia a través del cambio teórico constituyen problemas ya clásicos en la filosofía de la ciencia. Históricamente, desde diversas posiciones realistas se ha pretendido ofrecer respuesta a la tesis de la inconmensurabilidad apelando a la persistencia de clases y entidades teóricas a pesar de los cambios teóricos, y desde diversas posiciones antirrealistas se ha puesto en cuestión tal persistencia. Los problemas emergentes de dicho debate se han recuperado en discusiones recientes en el campo de la filosofía de la química ( $c f r$. Chang 2011), dado que se considera que la química ofrece los ejemplos paradigmáticos de clases naturales: los elementos y los compuestos químicos.

El problema de las clases ha aparecido casi exclusivamente entrelazado con la cuestión de la permanencia a través del cambio teórico, esto es, desde una perspectiva diacrónica. Y, por lo general, las estrategias argumentativas que defienden la existencia y persistencia de las clases naturales (o su inexistencia y no persistencia) parten de una consideración general de lo que las clases son en filosofía, para analizar luego qué dificultades presenta la química para la noción filosófica de clase natural, en particular, para el microestructuralismo esencialista, una concepción muy importante en filosofía de la química.

En este trabajo nos proponemos trastornar el tratamiento habitual del problema en dos sentidos. En primer lugar, nos acercaremos a las clases naturales desde una perspectiva sincrónica. Dejaremos de lado la perspectiva diacrónica porque creemos que ha nublado el tratamiento del problema, subordinando la pregunta por las clases a la defensa del realismo o el antirrealismo. La pregunta por la persistencia de las clases es, en todo caso, subsidiaria de la pregunta por la definición y la existencia misma de las clases naturales. En segundo lugar, abordaremos el problema de las clases naturales a partir de la consideración de tres problemas que presenta la química:

a) Si bien el número atómico permite identificar un elemento químico, no provee información químicamente relevante y, así, pasa por alto distinciones centrales en química.

b) La mecánica cuántica no da cuenta de la estructura molecular, propiedad de las moléculas que permite explicar muchas propiedades macroscópicas de las sustancias químicas. 
c) Las propiedades microscópicas no permiten definir unívocamente ciertas propiedades centrales para la química, como las correspondientes al par ácido-base.

Evaluaremos en qué medida las consecuencias que arroja el análisis de estos problemas ponen en cuestión la noción de clase natural. Para ello, en la sección $\S 2$ recordaremos el problema de las clases naturales tal como se ha planteado en filosofía. La sección $\S 3$ se ocupará del modo que la discusión filosófica ingresó en el ámbito de la filosofía de la química, en relación con las nociones de elemento y sustancia. En la sección $\S 4$ analizaremos los tres problemas mencionados, propios de la disciplina, a la luz de la cuestión de las clases naturales. En la sección $\S 5$ extraeremos las conclusiones del análisis, considerando si debe abandonarse la noción de clase natural en química o si tiene sentido mantenerla y, en tal caso, con qué noción de clase natural cabe comprometerse. Por último, en la sección $\S 6$ formularemos nuestras conclusiones generales y sus posibles consecuencias para la filosofía general de la ciencia.

\section{La concepción filosófica de las clases naturales}

La reflexión filosófica clásica sobre los términos generales del lenguaje natural ingresa en el ámbito propio de la filosofía de la ciencia cuando se aborda la cuestión de los términos que refieren a clases naturales postuladas por las teorías científicas. Una de las diferencias entre los términos denominados "teóricos" y los términos "de clase" es que los referentes de estos últimos, las clases naturales, pueden ser individuos observables (los miembros de la clase), lo que no generaría dificultad alguna para establecer la referencia del término. No obstante, según algunos autores, un término de clase pretende nombrar la clase y no el conjunto de individuos que la forman, de modo que, aun en el caso en que tales individuos son observables, es necesario explicar la manera en que los términos refieren a las clases (cfr. Psillos 1999). La búsqueda de una explicación del mecanismo referencial de este tipo de términos en ocasiones presupone la pregunta ontológica de qué son las clases naturales.

Cabe recordar que la discusión filosófica acerca de las clases naturales en filosofía de la ciencia se ha desarrollado entre dos posiciones extremas enfrentadas: el convencionalismo o psicologismo, inspirado en la filosofía de David Hume, y el esencialismo ( $c f r$. Córdoba y Lombardi 2012). De acuerdo con el primero, las clases naturales no son más que agrupamientos convencionales, muy útiles en ciencia, 
pero que no reflejan nada existente en la realidad; por lo tanto, si es necesario, las clasificaciones utilizadas en determinado contexto pueden modificarse en un contexto diferente. El esencialismo asume, por el contrario, la idea de que las clases naturales están realmente demarcadas en la realidad en la medida en que responden a esencias reales.

Bajo la influencia del empirismo, como el de Ernst Mach (1984 [1886]), que modeló el pensamiento del Círculo de Viena, la filosofía de la ciencia de la primera mitad del siglo XX adoptó, en general, posiciones más cercanas a la filosofía de Hume que al esencialismo. Estas posiciones se enfrentaron con el problema de que no lograban justificar por qué las leyes científicas permiten realizar predicciones exitosas. Mermada ya la influencia de las concepciones provenientes del Círculo de Viena, el esencialismo ganó adeptos en las décadas de 1970 y de 1980, asumiendo fuertes compromisos ontológicos en oposición a las concepciones humeanas tradicionales.

Como señalábamos, en las discusiones acerca del problema lingüístico de los términos de clase ha logrado imponerse el abordaje de la cuestión ontológica de qué son las clases naturales. Por ejemplo, John Dupré $(1993,2001)$ y Brian Ellis (2008) consideran que, para elucidar el problema filosófico de las clases naturales, es necesario comprender la distinción establecida por John Locke (1980 [1690]) entre esencia real y esencia nominal. Las esencias reales son la supuesta constitución interna de las cosas; no coinciden necesariamente con los rasgos perceptibles que asociamos con las cosas de una determinada clase. Las esencias reales, en cuanto postuladas y desconocidas, no desempeñan ningún papel en nuestra formación de conceptos. Las esencias nominales, en cambio, son los aspectos que provienen de la experiencia, que obtenemos a través de los sentidos y que convencionalmente asociamos con los tipos de objetos. Se supone que estos aspectos están vinculados con la constitución interna, real, de las cosas — con su esencia real-, pero esto es sólo una suposición. Esta distinción ha encontrado reformulaciones en la filosofía contemporánea, que en lugar de referir a esencias reales, suele referir a la naturaleza propia de los miembros de una clase determinada. Esta naturaleza se considera responsable de que estos miembros pertenezcan a dicha clase y no a otra. La esencia nominal está constituida por aquellos aspectos por medio de los cuales reconocemos los objetos pertenecientes a una determinada clase. Se suele asociar la esencia real con algún aspecto de la estructura microscópica, que sería la responsable de las propiedades macroscópicas o estructurales de los miembros de la clase. En contra del escepticismo lockeano, ciertos filósofos actuales han 
afirmado que la física y la química han descubierto las microestructuras en muchos ámbitos de lo real. Este optimismo, según Dupré, es el que manifestaban claramente Saul Kripke $(1972,1980)$ y Hilary Putnam $(1973,1975)$, filósofos ineludibles cuando se trata la cuestión de las clases naturales en ciencia.

Según Kripke y Putnam, las clases naturales son conjuntos de objetos definidos por la posesión común de propiedades teóricamente importantes, generalmente microestructurales. De acuerdo con el esencialismo de ambos autores, las clases naturales están determinadas por verdaderas esencias reales, en sentido lockeano; por lo tanto, la extensión de un término de clase está dada por la esencia real microestructural. La teoría causalista de la referencia, formulada por Kripke y Putnam, pretende explicar en qué consiste el mecanismo referencial de los términos de clases. La idea fundamental es que la naturaleza real de la esencia de la clase (recogida en el conocimiento experto por medio de métodos científicos apropiados, según Putnam) es aquello que determina la extensión del término. Tal como afirma Dupré, esta idea descansa en una fuerte presuposición ontológica: "la intención de referir a la naturaleza real preexiste a la caracterización de esa naturaleza" (Dupré 2001, p. 315).

La concepción causalista de la referencia defendida por Kripke y Putnam se opone al descriptivismo. El debate entre descriptivismo y causalismo en torno al funcionamiento de los términos excede los propósitos de este trabajo, pero cabe señalar que de acuerdo con la teoría descriptivista de la referencia - formulada por Gottlob Frege (1973 [1892]) y adoptada por Bertrand Russell (1905), John Searle (1958) y Peter F. Strawson (1959) con diferentes variaciones-, el significado de un nombre propio involucra dos dimensiones: la referencia o denotación y el sentido o connotación. La dimensión de la referencia o denotación es el objeto o entidad extralingüística denotada por el término en cuestión: el referente del término. El sentido, por su parte, es definido por Frege como el modo en el cual el referente se presenta. La dimensión del sentido está constituida por la descripción de cierta propiedad que permite identificar el objeto, permite determinar la referencia del término. El sentido de un término es, así, una descripción definida asociada con el término. Es el sentido (connotación) de un término lo que determina su referencia (denotación). Según esta concepción de la referencia, un término refiere a un objeto si y sólo si la descripción asociada con el término (el sentido) es verdadera respecto de ese objeto y no de otro. Hay un aspecto epistémico que resulta fundamental en la determinación de la denotación: el éxito referencial depende de cuánto sabe la comunidad 
lingüística competente acerca de un determinado individuo real, conocimiento que se recoge en la descripción definida o sentido. Ahora bien, de acuerdo con Frege, todos los signos del lenguaje funcionan como los nombres propios: tienen connotación y denotación. En el ámbito de la filosofía de la ciencia, algunos filósofos realistas toman esta teoría para su extrapolación al lenguaje científico. Respecto de los términos de clase, el descriptivismo sostiene que el significado de un término de clase está dado por una descripción que satisfacen todos y cada uno de los miembros de la clase, pero solamente ellos.

Kripke se opone al descriptivismo al afirmar que la relación que los términos tienen con sus objetos denotados es una relación directa, no mediada por ninguna otra instancia. Que un objeto determinado constituya el referente de un término se explica apelando a un vínculo causal que determina la referencia. La relación causal tiene lugar en el mecanismo de fijación de la referencia de un término, el "bautismo inicial" o "evento introductorio", y en una cadena causal, situada históricamente, que constituye el mecanismo de transmisión del término entre los hablantes. Según Kripke, los términos del lenguaje que se comportan de este modo son los denominados "designadores rígidos": nombres propios, términos de clases naturales y algunas descripciones matemáticas. Un designador rígido designa o refiere al mismo objeto en todo mundo posible en el que el objeto existe. Los designadores rígidos y las descripciones funcionan de modos diferentes: aquéllos garantizan la permanencia de la referencia a través de los mundos posibles, mientras que las descripciones sólo garantizan la permanencia de la referencia en tanto y en cuanto el objeto satisfaga las propiedades recogidas en la descripción. Los designadores rígidos, entonces, denotan directamente sus referentes, sin que medie en esta relación la atribución de ninguna propiedad. A diferencia de lo que ocurría con los nombres propios para Frege, los designadores rígidos no presentan una dimensión epistémica: refieren directamente a sus denotados sin que sea necesaria ninguna descripción verdadera para la apropiada relación referencial entre término y referente. Que la naturaleza del vínculo referencial aparezca despojada de todo aspecto epistémico posibilita que todas las creencias que asociemos con un término sean falsas, situación que en nada modifica aquel vínculo entre término y objeto, que se mantiene inalterable.

En principio, de acuerdo con el esencialismo, puede haber descripciones de los miembros de una clase que sean verdaderas respecto de ellos, y puede ocurrir que algunas descripciones recojan propiedades que dependen de las propiedades esenciales de los miembros de la 
clase. Aun así, este tipo de términos refieren a la esencia real microestructural, sin que sea necesaria ninguna descripción verdadera de los miembros de la clase. Efectivamente, las descripciones podrían ser falsas, y aun así, la referencialidad no corre peligro. Esto es así porque las descripciones no forman parte del significado de los términos de clase, no son las responsables de determinar la referencia de los términos.

Kripke y Putnam adoptan un fuerte compromiso ontológico, y también lo hacen aquellos filósofos que pretenden responder a la tesis de la inconmensurabilidad a través de la continuidad referencial en el cambio teórico. Sólo la confianza en un fuerte vínculo causal, que se funda sobre una necesidad de re (Ellis 2008), una necesidad que está en las cosas mismas, puede garantizar que un término que aparece en contextos teóricos distintos refiera a lo mismo a pesar del cambio. En este sentido, las esencias kripkeanas y putnamianas vienen a cumplir esa función: en la medida en que las esencias microestructurales sean las que determinen la referencia de un término de clase - ya sea independientemente de las creencias que asociemos con ellas (como afirmaría Kripke), ya sea que estas esencias sean recogidas por el conocimiento experto (como afirma Putnam) — y en la medida en que estas esencias sean independientes de nuestro conocimiento, porque están ahí, en el mundo, a la espera de que las descubramos, sólo así es posible que los términos de clase pertenecientes a teorías científicas sucesivas en el tiempo refieran siempre a las mismas clases, pese a la variación teórica. Se considera que son las cosas mismas, en última instancia, las responsables del éxito referencial.

Dupré critica el esencialismo, atendiendo a la ciencia real: afirma que el punto de vista "neoaristotélico" de Kripke y Putnam no puede sostenerse en la práctica científica. Por ejemplo, en biología, la unidad básica de clasificación es la especie. Ya sea que las especies formen clases naturales con esencias reales o no, ningún biólogo haría una afirmación tal para grupos de nivel más alto (las taxas), como géneros, familias, etc. La comprensión científica actual no brinda apoyo a una posición esencialista (Dupré 1993, véase también Hull 1965). Según el autor, en biología no sólo es problemático ofrecer una clasificación unívoca de las especies; la idea de que éstas son demarcadas por alguna propiedad interna necesaria y suficiente es antitética a todas las concepciones posdarwinianas de las especies. El esencialista podría responder que ello se debe a una imposibilidad de dicto (a un defecto de nuestro conocimiento) y no de re, ya que las esencias expresan una necesidad real. Pero Dupré subraya que la imposibilidad de identificar aquella propiedad interna para la caracterización de las especies se 
debe al reconocimiento de la omnipresencia de la variación, reconocimiento que todas las concepciones posdarwinianas integran en sus marcos teóricos. De modo que la idea de que no se ha hallado aún la propiedad esencial de cada especie biológica, y que será hallada oportunamente, no constituye más que una esperanza fundada en un supuesto esencialista ajeno a la práctica de la ciencia. Sobre esta base, Dupré propone abandonar todo intento de clasificaciones rígidas, y enfatiza la necesidad de múltiples y diferentes sistemas clasificatorios que pueden superponerse e intersectarse (Dupré 2001).

\section{El problema de las clases naturales en química}

Si bien pueden encontrarse casos de supuestas clases naturales en todas las disciplinas científicas, durante los últimos tiempos la química ha reemplazado a la biología como ciencia que suministra los casos paradigmáticos de clases naturales: los elementos químicos y sus compuestos. Se considera que el hecho de que un determinado ítem sea hierro explica su comportamiento y la predictibilidad de tal comportamiento. Así, el hecho de que los objetos de hierro sean magnetizables es la expresión de una "ley de la naturaleza". Asimismo, el hierro es claramente diferente de sus vecinos en la tabla periódica (manganeso y cobalto) y no existe ningún elemento que conforme una clase intermedia.

En química, la pregunta acerca de la existencia de clases naturales está estrechamente relacionada con el problema del microestructuralismo esencialista que, en este ámbito, supone que los elementos y las sustancias químicas se individualizan exclusivamente por su microestructura: "Es esencial al hierro que algo hecho de hierro puro esté constituido por átomos que tienen precisamente 26 protones en su núcleo" (Bird y Tobin 2016, introducción de la sección §2.2). El microestructuralismo esencialista parecería funcionar con los elementos químicos, puesto que el número atómico $\mathrm{Z}$ (número de electrones del átomo neutro o de protones en el núcleo) resultaría suficiente para individualizar cada elemento.

La utilización de $\mathrm{Z}$ para identificar la clase natural-elemento ${ }^{1}$ puede rastrearse hasta la discusión en torno de la denominada "crisis de los isótopos". La clasificación periódica, basada originalmente en los pesos atómicos de los elementos, enfrenta una crisis a comienzos del siglo XX, cuando se descubre una gran cantidad de isótopos, esto es, átomos de lo que se consideraba un mismo elemento, pero con

${ }^{1}$ En relación con el debate acerca de la definición de elemento químico, $c f r$. van der Vet 1979; Hendry 2006, y Schwarz 2007. 
pesos atómicos diferentes y propiedades macroscópicas ligeramente diferentes. Si se considera que los elementos son sustancias que pueden aislarse en la práctica, la multiplicidad de isótopos parecía indicar una repentina multiplicación de "elementos"; esto condujo a varios químicos a proclamar la desaparición de la tabla periódica como tal y su reemplazo por la tabla de los isótopos (Scerri 2005). Frente a ello, Friedrich Paneth retoma la distinción introducida por Mendeleev entre sustancia simple y sustancia básica: la sustancia simple es una entidad empírica, que puede manipularse y que posee propiedades observables; la sustancia básica, por el contrario, es una entidad teórica, constituyente última de la materia, que se preserva a través de las transformaciones químicas, y cuya única propiedad es el número atómico Z (cfr. Córdoba, Labarca y Zambon 2013). Esta distinción filosófica ejerció un papel crucial para la supervivencia de la tabla periódica como tal: sobre la base de los trabajos de Paneth, la IUPAC (International Union of Pure and Applied Chemistry) modificó en 1923 la propiedad definitoria de los elementos químicos, pasando del peso atómico al número atómico ${ }^{2}$. Si se ontologiza la recomendación de la IUPAC, $Z$ se constituye como la propiedad esencial, identificatoria de las clases naturales en química. Sin embargo, como veremos en la próxima sección, la postura que identifica un elemento químico como clase natural a través de su número atómico se enfrenta con diversas dificultades, relacionadas con la especificidad de la propia química.

Si el microestructuralismo se enfrenta ya con algunos inconvenientes en el caso de la identidad de los elementos químicos, cuando se lo intenta extender a los compuestos aparecen dificultades adicionales. Se ha cuestionado el ejemplo paradigmático de los argumentos de Kripke y Putnam: "agua es $\mathrm{H}_{2} \mathrm{O}$ ", porque los compuestos como el agua tienen una estructura esencialmente dinámica, que no puede ser adecuadamente descrita por una fórmula química: los elementos componentes no son suficientes para dar cuenta del compuesto ( $c f r$. Needham 2000; van Brakel 2000; Weisberg 2005). De acuerdo con Robin Hendry (2006), cualquier muestra de agua, aun cuando se trata de agua pura, contiene no sólo moléculas de $\mathrm{H}_{2} \mathrm{O}$, sino también iones $\mathrm{H}_{3} \mathrm{O}^{+}{\text {y } \mathrm{OH}^{-}}^{-}(c f r$. discusión en Córdoba y Lombardi 2012). A partir de esto, no sería estrictamente correcto afirmar que el agua es "esencialmente $\mathrm{H}_{2} \mathrm{O}$ "; debería afirmarse que no es únicamente $\mathrm{H}_{2} \mathrm{O}$

\footnotetext{
${ }^{2}$ Paneth fue reconocido principalmente por la reintroducción de la distinción entre sustancia básica y sustancia simple en las conferencias de Königsberg de 1931, reeditadas en Paneth 1962.
} 
o, más bien, que "el agua es $\mathrm{H}_{2} \mathrm{O}$ y también es realmente otras cosas" (Chang 2012a, p. 203).

Si bien los problemas mencionados se han discutido en filosofía de la química, no han logrado resolverse y su abordaje no suele ofrecer alternativas para pensar las clases naturales. Algunas posiciones afirman que es necesario abandonar el microesencialismo desde posturas antirreduccionistas (Lombardi y Labarca 2005), mientras otras continúan defendiendo el microesencialismo sobre la base de suponer la reducción de la química a la física ( $c f r$. por ejemplo, Hettema 2012). En lo que sigue, analizaremos tres problemas provenientes del campo de la química.

\section{Tres problemas particulares de la química}

Como se ha señalado, tanto en la filosofía general de la ciencia como en la filosofía de la química, las propiedades microscópicas se consideran los factores responsables de las propiedades macroscópicas de los elementos químicos y de los compuestos. Este supuesto está en la base de diferentes posturas microesencialistas: la estructura microscópica es esencial porque determina las restantes propiedades de la especie química. De este modo, en química se lograría una armoniosa compatibilidad entre la esencia real y la esencia nominal lockeanas.

Si bien se han brindado argumentos en contra de aquel supuesto en el contexto de la discusión sobre las relaciones entre química y física, aquí nos enfocaremos en tres problemas específicos. El propósito es analizarlos en el marco de la pregunta por la existencia de clases naturales en química y determinar sus consecuencias para el microestructuralismo, con vistas a extraer conclusiones filosóficas más generales acerca de las clases.

\subsection{El problema de la identidad de los elementos químicos}

Como se señaló, la versión más frecuente del microesencialismo considera el número atómico $\mathrm{Z}$ como la esencia de los elementos químicos. De acuerdo con la IUPAC, Z representa el "número de protones del núcleo atómico" y un elemento químico se define como una sustancia que posee "todos los átomos con el mismo número de protones en el núcleo atómico" (IUPAC Golden Book). Esta caracterización de los elementos químicos es usual en química cuántica, donde las sustancias macroscópicas se modelan como un conjunto de átomos con la misma estructura interna compuesta por un núcleo y un conjunto de electrones. Independientemente de que tal elección pueda resultar un criterio clasificatorio conveniente en la práctica, en el presente 
contexto la pregunta es si el número atómico constituye la propiedad esencial que permite individualizar un elemento químico como clase natural.

En primer lugar, cabe recordar que las propiedades específicamente químicas de los elementos, en particular su capacidad de reaccionar con otros elementos, no dependen del núcleo de sus átomos, sino de sus electrones. Por lo tanto, en modo alguno parece "natural" que la esencia de una clase natural química dependa del número de las partículas que no juegan un papel relevante en el comportamiento de los miembros de la clase.

El microesencialista defensor del número atómico $\mathrm{Z}$ como propiedad esencial podría replicar que el número de protones es el mismo que el número de electrones, por lo cual adoptar el número $\mathrm{Z}$ como propiedad esencial también considera el número de electrones. Sin embargo, la igualdad entre el número de protones y el número de electrones sólo se cumple en los átomos eléctricamente neutros. Los elementos químicos también pueden presentarse bajo la forma de partículas cargadas denominadas iones, que poseen más electrones (iones negativos o aniones) o menos electrones (iones positivos o cationes) que los que posee el átomo eléctricamente neutro. La presencia de iones de los elementos químicos no es una situación excepcional: por el contrario, es difícil encontrar muestras de elementos químicos en solución o en fase gaseosa sin la presencia de iones. Por otra parte, los iones de ciertos elementos, como el sodio, el potasio y el calcio, desempeñan un papel protagónico en la biología molecular de los organismos, ya que de su característica iónica depende su comportamiento frente a las membranas celulares. Frente a ello, no es claro por qué especies químicas de comportamientos muy diferentes deben considerarse miembros de una misma clase natural definida por una propiedad, el número atómico, que no se relaciona con tales comportamientos.

Pero aun en el caso de los elementos que se presentan bajo la forma de átomos eléctricamente neutros, el número $\mathrm{Z}$ parece no ser suficiente para individualizar clases naturales químicamente relevantes. Mientras que el número $\mathrm{Z}$ es una propiedad de los átomos, las propiedades químicas de los elementos dependen no de sus átomos, sino de las moléculas que tales átomos conforman. La alotropía es la propiedad que poseen algunos elementos químicos, definidos en términos de Z, de formar agregados que contienen los mismos tipos de átomos pero que presentan propiedades macroscópicas radicalmente diferentes. Por ejemplo, el oxígeno puede presentarse como oxígeno 
atmosférico y como ozono, el fósforo se presenta como fósforo rojo y fósforo blanco. El caso típico de alotropía es el del carbono, de número atómico $Z=6$, que se puede aislar o sintetizar empíricamente como grafito, diamante, grafeno y fullereno, especies químicas de propiedades y comportamientos macroscópicos totalmente disímiles. En estas diferentes presentaciones, la molécula de carbono posee estructuras moleculares distintas: si bien en todos los casos $\mathrm{Z}=6$, los átomos del elemento carbono están unidos en la molécula de manera completamente diferente. En el caso del diamante, la molécula de carbono tiene una estructura espacial tetraédrica $\left(\mathrm{sp}^{3}\right)$; en el caso del grafito, la disposición espacial de los átomos es hexagonal en capas superpuestas $\left(\mathrm{sp}^{2}\right)$. Si el comportamiento macroscópico del elemento depende de la estructura espacial de sus moléculas, el número atómico de los átomos en modo alguno resulta suficiente para distinguir las distintas estructuras espaciales en las que las moléculas se presentan. El caso del carbono pone de manifiesto, además, no sólo las limitaciones del número atómico para asociarse con propiedades macroscópicas, sino que ni siquiera puede suponerse que en algún momento se contará con una descripción macroscópica acabada de los elementos químicos: grafeno y fullereno son manifestaciones del carbono muy recientemente descubiertas, con propiedades completamente inesperadas, cuyas aplicaciones parecen abrir un nuevo y fructífero campo tecnológico.

La identificación de los elementos químicos mediante su número atómico deja de lado la consideración del conjunto de los electrones del átomo; incluso deja de lado aquellos electrones que tienen funciones de conexión, esto es, lo que se denomina la estructura electrónica de los átomos. Las estructuras electrónicas y sus variaciones cumplen un papel central en la explicación de algunas de las propiedades de las sustancias macroscópicas en el laboratorio químico. Y esto sí constituye un auténtico desafío para el microestructuralismo: cuando se considera que es $\mathrm{Z}$ el único criterio de identificación de un elemento como clase natural, se está dejando de lado todo aquello que resulta relevante para la explicación de las propiedades químicas de los elementos y sus compuestos. Por ejemplo, si no se toman en cuenta los electrones del átomo, no es posible dar cuenta de por qué un elemento se puede presentar bajo diferentes formas alotrópicas. ${ }^{3}$ En definitiva, cuando se considera que el número atómico está asociado

${ }^{3}$ Es necesario establecer la relación entre $Z$ y la identificación de un elemento químico. Esto se requiere debido a que las mediciones del número atómico no se realizan por medios "químicos", sino usando una interpolación de valores por medio de mediciones generalmente espectroscópicas. En ese sentido, constituye una 
al núcleo y rara vez éste es objeto de estudio de la química, la consagración del número atómico como propiedad esencial del elemento químico como clase natural debería, al menos, resultar sospechosa.

Frente a estos inconvenientes, el microestructuralista podría reconocer las limitaciones del número atómico para su proyecto y cambiar de propiedad microestructural esencial para individualizar los elementos como clases naturales. En particular, podría considerar la configuración electrónica como propiedad definitoria, ya que de ella depende en gran parte el comportamiento químico de los elementos. No obstante, la configuración electrónica específica de un elemento no implica la manifestación de un comportamiento químico característico en el nivel macroscópico, no determina sus macro-propiedades ( $c f r$. Scerri 1997): por ejemplo, si bien el helio, el berilio y el magnesio tienen la misma configuración de los electrones de la capa externa, el helio es un gas altamente inerte, mientras que el berilio y el magnesio son metales reactivos.

El microestructuralista podría aún replicar que su postura requiere la consideración de la microestructura completa del átomo: no sólo su número atómico, sino también la distribución completa de sus electrones y no sólo los de la última capa. En este caso, la microestructura no toma en cuenta los neutrones del núcleo. Si bien es cierto que muchas propiedades químicas no dependen de los neutrones, éstos con centrales en las explicaciones radioquímicas de la estructura interna de los isótopos de un mismo elemento y sus series de decaimiento radioactivo. Tradicionalmente las propiedades de los elementos radioactivos no han sido tenidas en cuenta en la discusión acerca de la microestructura de un elemento. Sin embargo, deberían ser seriamente consideradas, dado que las clasificaciones químicas son mucho más ricas y complejas de lo que suele suponerse habitualmente: si bien los elementos químicos suelen ser pensados como los "ladrillos" con los que se construyen otros materiales, la tabla de radioisótopos es tan frecuente como la tabla periódica misma.

En definitiva, si se decide ser microestructuralista respecto de la individualización de las clases naturales en química, ¿qué se entiende por "microestructura"?, ¿el número atómico, la configuración electrónica del átomo, la estructura completa del átomo, incluyendo sus neutrones, la estructura espacial de la molécula? Estas preguntas ponen de manifiesto que la clasificación en química, incluso la que

propiedad diferente de aquellas que constituyen ejemplos estándar de propiedades químicas, como ser inflamable, ser ácido o ser un agente reductor, es decir, propiedades susceptibles de ser medidas usando reacciones químicas. 
parece menos controversial, como la referida a los elementos, es una cuestión problemática. Y esta problematicidad excede el tratamiento habitual de la filosofía general de la ciencia respecto de las clases naturales.

El énfasis en la microestructura como aquello que brinda, de un modo u otro, la propiedad esencial para identificar un elemento químico como clase natural, implícita o explícitamente se basa en el supuesto de la reducción ontológica de la química macroscópica a la química molecular, y de ésta a la física, en particular, a la mecánica cuántica. Sin embargo, la posición de considerar los elementos como clases naturales no necesita encontrarse conceptualmente ligada a supuestos reductivos o microestructuralistas. En particular, es posible encontrar en el ámbito macroscópico otros candidatos para identificar un elemento químico. Por ejemplo, Mark Leach (2013), discutiendo la dimensionalidad de la tabla periódica y las razones por las cuales ésta es presentada en la forma corta estándar, postula la electronegatividad como propiedad esencial microestructural de los elementos como clases naturales. Por otra parte, un candidato a propiedad esencial no microestructural es el llamado punto triple en termodinámica, esto es, el estado termodinámico (definido por la temperatura y la presión del sistema) en el cual coexisten en equilibro el estado sólido, el estado líquido y el estado gaseoso de una sustancia: el punto triple es específico de cada sustancia y la individualiza (Needham 2008, van Brakel 2012; para una discusión, cfr. Córdoba, Labarca y Zambon 2013). Además de ser una propiedad macroscópica de más fácil acceso experimental que cualquier propiedad microestructural, el punto triple cuenta con la ventaja de ser conceptualmente aplicable a cualquier sustancia química (si bien no siempre con la misma accesibilidad empírica), y no sólo a los elementos. Si se contara con una propiedad microestructural que pudiera oficiar de propiedad esencial para identificar un elemento químico como clase natural, podría argumentarse en favor de asumir una perspectiva microestructuralista; pero dado que el microestructuralismo no carece de problemas, no parece existir un motivo filosófico suficiente para buscar la propiedad esencial de la clase en el ámbito microscópico y no en el ámbito macroscópico.

\subsection{El problema de la estructura molecular}

Las dificultades para concebir los elementos químicos como clases naturales en términos microestructurales se agudizan cuando se trata de extender el microestructralismo a las sustancias químicas en general. 
Como fue señalado en la subsección anterior, la postura reduccionista suele suponer la reducción ontológica de la química macroscópica a la química molecular, y de ésta a la mecánica cuántica. El microestructuralista podría, entonces, considerar que la propiedad esencial identificatoria de las sustancias es la estructura molecular, bajo el supuesto de que dicha estructura determina las propiedades macroscópicas de la sustancia. Sin embargo, esta estrategia cuenta con sus propias dificultades específicas, relacionadas con las peculiaridades de la mecánica cuántica. A continuación presentaremos y analizaremos tales dificultades.

En química, la composición de una molécula generalmente está dada por su fórmula empírica, en la que se detallan sus elementos constituyentes y en qué proporción estequiométrica se los encuentra. Esta fórmula, sin embargo, no brinda información acerca de la disposición geométrica de tales componentes. Por ejemplo, la fórmula $\mathrm{C}_{2} \mathrm{H}_{4} \mathrm{O}_{2}$ puede corresponder a diferentes compuestos químicos: el formiato de metilo, el ácido acético y el glicolaldehído. Los compuestos que contienen la misma cantidad de átomos (es decir, poseen la misma fórmula empírica) pero que difieren en su disposición espacial se denominan isómeros. El isomerismo es un fenómeno particularmente importante en química, pues permite explicar la diferencia en los comportamientos químicos y físicos de sustancias con la misma composición. Los isómeros se dividen en dos grandes grupos. Los isómeros estructurales son aquellos que contienen la misma cantidad y tipo de átomos en su fórmula empírica, pero difieren en la manera en que están interconectados sus átomos constituyentes. Por otra parte, los isómeros que contienen la misma cantidad y tipo de átomos y, además, las mismas conexiones interatómicas, se denominan estereoisómeros, y sólo difieren en la distribución espacial de sus átomos. Dentro de este grupo nos detendremos en los llamados isómeros ópticos: los miembros de un par de isómeros ópticos son imágenes especulares que no pueden superponerse, de modo análogo a nuestras dos manos. La propiedad que diferencia a los miembros de un par de isómeros ópticos se denomina quiralidad. Las moléculas que presentan quiralidad tiene una función relevante dentro de las reacciones enzimáticas de los sistemas biológicos: una gran cantidad de fármacos son quirales y generalmente sólo uno de ellos exhibe actividad biológica.

La peculiaridad de los isómeros ópticos de un mismo compuesto es que comparten casi todas sus propiedades químicas y físicas, pero se diferencian entre sí por el tipo de interacción que manifiestan con la luz polarizada. En efecto, hay una relación directa entre la actividad 
óptica de una sustancia y la presencia de un centro de quiralidad: los isómeros ópticos tienen la propiedad de girar el plano de polarización de la luz polarizada cuando ésta atraviesa estas moléculas. Si el plano de polarización gira hacia la derecha, se dice que el isómero óptico es dextrógiro (D); si lo hace hacia la izquierda, se denomina levógiro (L). Los isómeros D y L de una sustancia quiral se denominan enantiómeros: siempre giran la luz en la misma medida, pero en direcciones opuestas. En una mezcla equimolar de dos enantiómeros, la rotación neta es cero: las mezclas de este tipo se denominan racémicas. Suele observarse que, en un compuesto ópticamente activo, con el tiempo la mitad de las moléculas tienden a adquirir quiralidad opuesta de modo de igualarse el número de moléculas levógiras y dextrógiras; este proceso por el cual el compuesto se convierte en ópticamente neutro se denomina racemización.

Ahora bien, cuando se pretende brindar la descripción mecánicocuántica de una molécula, el operador hamiltoniano, que permite calcular los valores posibles de energía para estas moléculas, sólo depende de las distancias entre las partículas que la componen. En el caso de isómeros estructurales de un mismo compuesto, la diferencia entre ellos se manifestará en el propio hamiltoniano y, con ello, en sus energías. Pero, ¿qué sucede con los isómeros ópticos? En este caso, las distancias interatómicas son iguales para los dos miembros del par de enantiómeros, de modo tal que el hamiltoniano es exactamente el mismo para los dos miembros. En consecuencia, la mecánica cuántica brinda exactamente la misma descripción para dos estructuras químicas que pueden efectivamente ser diferenciadas en la práctica a través de su actividad óptica, incluso por sus propiedades químicas en reacciones que son estereoespecíficas.

Es importante resaltar que este problema que presentan los isómeros ópticos es por completo independiente de la aproximación Born-Oppenheimer, en particular, de la aproximación de núcleo fijo (clamped-nucleus approximation), que consiste en postular una estructura dada por las posiciones fijas de los núcleos en el espacio, tal como se supone en la química estructural (no cuántica) que se ocupa de la descripción geométrica de las moléculas (para una discusión de esta aproximación, $c f r$. Lombardi y Castagnino 2010). Supongamos, por ejemplo, que contáramos con el hamiltoniano exacto $H$ (sin aproximación alguna) de una molécula de alanina $\mathrm{C}_{3} \mathrm{H}_{7} \mathrm{NO}_{2}$, que incluye tres núcleos de carbono, siete de hidrógeno, uno de nitrógeno y dos de oxígeno, más todos sus electrones. Aun cuando no podamos escribir este hamiltoniano por su complejidad, sabemos que sólo dependerá de las distancias entre las partículas componentes. Por 
lo tanto, ni siquiera el hamitoniano exacto puede dar cuenta de la diferencia entre los miembros de un par de isómeros ópticos. Como afirman Brian Sutcliffe y Guy Woolley: "Entonces, jclaramente un autoestado del $H$ no corresponde a una molécula clásica con estructura! Esta observación plantea la pregunta: ¿Cuáles son las ecuaciones que determinan los estados cuánticos de una molécula? Más allá de la aproximación BO [Born-Oppenheimer] no tenemos idea" (Sutcliffe y Woolley 2012, p. 416). En definitiva, el problema de la distinción mecánico-cuántica entre enantiómeros es un problema que excede la aproximación Born-Oppenheimer y sus supuestos subyacentes; el problema apunta a una dificultad profunda en los intentos de dar cuenta de la química estructural en términos cuánticos. En otras palabras, conocer la estructura química y la posición del núcleo es imposible utilizando el modelado cuántico de un sistema químico. Los químicos cuánticos construyen sus supuestos iniciales acerca de la posición fija de los núcleos utilizando estructuras moleculares determinadas por razonamientos provenientes de la teoría estructural química; tales supuestos son ajenos a la mecánica cuántica (para una discusión sobre este tema, $c f r$. Fortin, Lombardi y Martínez González 2016).

El uso de estas nociones estructurales para describir las estructuras químicas constituye el mayor obstáculo para el microestructuralismo esencialista respecto de los compuestos químicos. Aun si se aceptara que la estructura molecular logra identificar a los elementos químicos como clases naturales, dicha clasificación natural no podría fundamentarse mediante la mecánica cuántica. En particular, si bien la diferencia entre los miembros del par de isómeros ópticos es estructural, la descripción mecánico-cuántica en términos de sus constituyentes no permite diferenciarlos: la mecánica cuántica dicta valores de energía iguales para estas sustancias, que en la práctica pueden ser aisladas y que poseen características físicas y químicas diferentes. Pero, desde una perspectiva microestructuralista, el ámbito cuántico debería ser el que explicara las diferencias estructurales y, en consecuencia, las diferencias en las propiedades químicas y físicas macroscópicas de las sustancias compuestas.

Esta situación sugiere las siguientes preguntas: ¿qué tan micro es una microestructura? Es cierto que la estructura molecular es una propiedad microscópica de las sustancias, pero la descripción mecánico-cuántica en términos del hamiltoniano de las moléculas también es microscópica, incluso de un nivel más microscópico que el anterior. Entonces, ¿en qué nivel microscópico deben buscarse las características que permiten agrupar las sustancias químicas como 
una clase? Si el nivel estructural y el nivel cuántico clasifican las sustancias de diferente modo, ¿cuál de los dos niveles posee la prioridad ontológica para determinar las clases naturales? En la medida en que el microestructuralismo esencialista no enfrente estos desafíos que le impone la química cuántica, su propuesta continuará mostrando un flanco débil a la luz de la práctica de la ciencia.

\section{3. ¿Qué hace que los ácidos sean ácidos?}

Hasta aquí hemos considerado dos casos en los que se manifiestan dificultades en la clasificación química, que involucran la noción de elemento químico y sustancia química. Sin embargo, no son éstas las únicas clasificaciones importantes en química. Por el contrario, existen otras propiedades que se han utilizado ampliamente para clasificar las sustancias químicas con importante poder explicativo. Éste es precisamente el caso de la clasificación ácido-base.

El par ácido-base ha sido por excelencia una clasificación tradicional y central de las sustancias químicas, que ha dominado la disciplina desde su consolidación como tal. Esta clasificación presenta casos paradigmáticos: el caso de los ácidos minerales, tales como el ácido clorhídrico $(\mathrm{HCl})$, el ácido nítrico $\left(\mathrm{HNO}_{3}\right)$ y el ácido sulfúrico $\left(\mathrm{H}_{2} \mathrm{SO}_{4}\right)$, cuyas contrapartes son las sustancias básicas, como los hidróxidos de los metales alcalinos $(\mathrm{NaOH} ; \mathrm{KOH})$. Las reacciones estándar entre estas sustancias se denominan reacciones de neutralización y dan lugar a la formación de otro grupo de sustancias denominadas sales y agua. La obtención del cloruro de sodio $(\mathrm{NaCl})$ puede ejemplificar este tipo de reacciones:

$$
\text { [ácido] } \mathrm{HCl}+\text { [base] } \mathrm{NaOH} \rightarrow \text { [sal] } \mathrm{NaCl}+\text { [agua }] \mathrm{H}_{2} \mathrm{O}^{4}
$$

Si bien este ejemplo muestra cómo se puede entender una reacción de neutralización entre un par ácido-base para formar sales y agua, la descripción de cuáles son las características que determinan el comportamiento de los ácidos está fuertemente ligada a su comportamiento macroscópico, que se expresa a través de una definición operacional de acidez. Dicha definición está asociada a los efectos corrosivos de los ácidos sobre los metales y a la posibilidad de identificarlos por medio de indicadores para la medición de variaciones del $\mathrm{pH}$. Incluso es interesante notar que la caracterización de los ácidos minerales (sulfúrico y nítrico) no es una caracterización teórica, en el sentido de que no se apela a ninguna teoría para caracterizarla. Por

\footnotetext{
${ }^{4}$ La notación entre corchetes no es propia de la terminología química: sólo se incluye en favor de la claridad de la exposición.
} 
el contrario, parece ser el caso que estas "clases estables" de ácidos han sido caracterizadas más por descripciones cotidianas que por un análisis de su microestructura, como sugiere Chang (2012). ${ }^{5}$

No obstante, y a pesar de la aparente unicidad de la clasificación ácido-base y su extendida utilización, ésta no ha podido ser descrita en términos microscópicos en el ámbito de una sola teoría. La presencia central de la diferencia entre ácidos y bases en química macroscópica contrasta con la compleja relación que existe entre las distintas teorías que pretenden dar cuenta de dicha diferencia desde una perspectiva microestructural.

La búsqueda del "principio" de la acidez preocupó a químicos de la talla de Lavoisier y Liebig desde los inicios de la química moderna. Svante Arrhenius fue el primero en reconocer, en la década de 1880, que las moléculas de agua pueden disociarse de manera espontánea en sus iones $\mathrm{H}^{+}$y $\mathrm{OH}^{-}$en concentraciones iguales. Para Arrhenius, en una solución acuosa ácida hay una mayor concentración de iones $\mathrm{H}^{+}$ que de $\mathrm{OH}^{-}$. De esta manera, identificó la acidez con la capacidad de una sustancia que contiene hidrógeno para producir un exceso de $\mathrm{H}^{+}$, y la basicidad (o alcalinidad), con la capacidad para producir iones $\mathrm{OH}^{-}$. En términos actuales, la acidez de Arrhenius puede ser descrita como la formación del ion hidronio $\left(\mathrm{H}_{3} \mathrm{O}^{+}\right)$y su posterior hidratación $\mathrm{H}_{5} \mathrm{O}_{2}^{+}$:

$$
\begin{gathered}
\mathrm{H}_{(a q)}^{+}+\mathrm{H}_{2} \mathrm{O}_{(\text {liq })} \rightarrow \mathrm{H}_{3} \mathrm{O}_{(a q)}^{+} \text {[ion hidronio] }{ }^{6} \\
\text { [ion hidronio] } \mathrm{H}_{3} \mathrm{O}_{(a q)}^{+}+\mathrm{H}_{2} \mathrm{O}_{(\text {liq })} \leftrightarrow \mathrm{H}_{5} \mathrm{O}_{2(a q)}^{+}
\end{gathered}
$$

En 1923, Johannes Brønsted y Thomas Lowry postularon una generalización del concepto de acidez de Arrhenius. Para estos autores, la acidez puede ser descrita como un proceso en el cual sólo están involucrados los iones $\mathrm{H}^{+}$, pero los iones $\mathrm{OH}^{-}$no intervienen en la definición de alcalinidad. En este marco, los ácidos son (al igual que en el caso de Arrhenius) donadores de $\mathrm{H}^{+}$, y las bases son receptores de $\mathrm{H}^{+}$. Desde esta perspectiva, entonces, el ion $\mathrm{OH}^{-}$es tan sólo un caso particular de un receptor de iones $\mathrm{H}^{+}$:

$$
\mathrm{H}^{+}+\mathrm{OH}^{-} \rightarrow \mathrm{H}_{2} \mathrm{O}
$$

\footnotetext{
${ }^{5}$ Agradecemos a uno de los árbitros por sugerirnos referir el caso de los ácidos minerales y su descripción.

${ }^{6}$ Los subíndices significan: $(a q)$, "en solución acuosa"; (liq), el estado de agregación "líquido".
} 
De manera simultánea pero independiente, también en el año 1923 Gilbert Lewis definió el par ácido-base de la siguiente manera: "una sustancia básica es la que tiene un par de electrones que pueden ser usados para completar el grupo estable de otro átomo, y una sustancia ácida es la que puede emplear un par [de electrones] libre de otra molécula para completar el grupo estable de unos de sus átomos. En otras palabras, la sustancia básica suministra un par de electrones para un enlace químico, la sustancia ácida acepta tal par" (Lewis 1923, p. 142). En este caso, los conceptos de acidez y alcalinidad están estrechamente asociados al concepto de enlace químico:

(ácido de Lewis) $\mathrm{A}+$ base de Lewis : $\mathrm{B} \leftrightarrow \mathrm{A}^{-}-\mathrm{B}^{+}$

(enlace covalente)

En la práctica, los químicos no encuentran dificultades en estas múltiples definiciones de acidez y alcalinidad, ya que cuentan con un conocimiento heurístico general que les permite decidir qué caracterización utilizar en cada caso. El problema es de carácter teórico: no existe una respuesta única a la pregunta acerca de cuáles son las características estructurales que otorgan a una sustancia su comportamiento ácido o básico. Como afirma claramente Hasok Chang: "la multiplicidad de miradas científicas acerca de qué tipos de objetos pueden poseer acidez creará un problema para aquellos que creen que el término 'ácido' selecciona un grupo de objetos con la misma constitución interna esencial" (2012b, p. 693). En otras palabras, este caso de clasificación en química presenta una nueva dificultad para una posición microestructuralista comprometida con la existencia de esencias.

\section{Un pluralismo a la medida de la química}

Los problemas analizados constituyen obstáculos importantes para considerar las clases naturales en química en el sentido habitual del microestructuralismo esencialista. ¿Qué debemos hacer con la noción de clase natural en esta disciplina? El modo de clasificar en química, en relación con las dificultades señaladas, ¿torna superflua la noción de clase?, ¿tiene ésta algún valor, heurístico, por ejemplo? ¿Es conveniente retener su uso? ¿O conviene dejar de utilizar el concepto, abandonar la idea de clases naturales en química?

Hemos señalado que, por lo general, estas discusiones culminan con la adopción del convencionalismo, fundado en una actitud filosófica estrictamente empirista, o del esencialismo, sobre la base de la adopción de fuertes compromisos ontológicos. En el caso particular 
de las clases en química, los problemas para el microestructuralismo parecen inclinar la balanza hacia el convencionalismo. Sin embargo, a pesar de las dificultades para hallar en las microestructuras la clave de las clasificaciones químicas, resulta difícil creer que tales clasificaciones responden exclusivamente a convenciones útiles, sin respaldo alguno en la realidad.

Frente a esta situación, es posible proponer otras alternativas para pensar las clases naturales desde una perspectiva filosófica. De hecho, se ha ofrecido una concepción respecto de las clases naturales que no cae en el convencionalismo pero que, a la vez, se desliga de todo esencialismo por medio de la adopción de un pluralismo ontológico que recupera la inspiración kantiana para la filosofía de la ciencia (cfr. Córdoba y Lombardi 2012). Este pluralismo, desarrollado por Olimpia Lombardi y Ana Rosa Pérez Ransanz (2008; 2012), ha mostrado su fecundidad para abordar problemas filosóficos como el de la reducción, en particular, aplicado a la relación entre química y física (cfr. Córdoba y Martínez González 2014). Si bien no continuaremos aquí esa línea, coincidimos con la posición de Lombardi y Pérez Ransanz en considerar que no cabe otra opción más que adoptar cierto pluralismo para pensar las clases en filosofía de la química.

Para pensar las clases en química desde la perspectiva de los problemas presentados, parece particularmente pertinente una propuesta reciente en filosofía de la ciencia: el pluralismo epistémico que desarrolla Hasok Chang. Aquí consideraremos dicho pluralismo, poniendo énfasis en el realismo activo del autor, en la idea de que la realidad es aquello que nos ofrece resistencia.

Si bien el pluralismo de Chang es epistémico y nada afirma respecto de cómo es el mundo al que la ciencia refiere, propone un giro en el modo de comprender el debate tradicional acerca del realismo científico: sostiene que la discusión sobre el realismo debe dejar de lado la referencia a la verdad, pero retomar la cuestión de la realidad.

¿Por qué debe abandonarse toda referencia a la verdad? De acuerdo con el autor, las teorías científicas no son verdaderas o falsas en el sentido de la correspondencia entre lenguaje y realidad. De hecho, Chang prefiere no hablar de teorías, sino de sistemas de práctica. Un sistema de práctica es "un conjunto coherente e interactivo de actividades epistémicas que se proponen alcanzar ciertos propósitos. Cada sistema encarna una explicación de un determinado aspecto de la realidad, que constituye su objeto de investigación, así como los métodos para crear y utilizar tales explicaciones" (Chang 2012a, p. 260). La elección de Chang de reemplazar la noción de teoría por 
la de sistema de práctica es consistente con su concepción del conocimiento, de acuerdo con la cual éste debe ser interpretado como habilidad y no como creencia. Considera que el "saber hacer" algo no requiere necesariamente saber proposicional, ni tampoco lo produce: "podemos saber cómo fundir hierro sin, de este modo, obtener ninguna idea precisa acerca de la temperatura a la que aquello ocurre" (Chang 2004, p. 150). El abandono de la idea tradicional de conocimiento como saber proposicional arrastra consigo la noción de verdad, dado que no tiene sentido predicar verdad o falsedad de una habilidad o práctica. En resumen, el conocimiento se interpreta como una habilidad para actuar sin frustrarse por la resistencia de la realidad.

Ahora bien, ¿qué entiende el autor por realidad? Chang se compromete con una "definición operacional" de la realidad externa: "Por 'realidad' quiero decir aquello que sea que existe 'allí afuera' ('out there'), que no puede ser controlado por la propia voluntad" (2012a, p. 217). El autor toma del pragmatismo la idea de que una de las más importantes fuentes de la noción misma de realidad es la resistencia de la naturaleza a nuestros planes. El compromiso con este realismo mínimo es toda la metafísica que Chang está dispuesto a admitir. Sobre esta base, se refiere a la realidad como aquello que, de algún modo, constriñe nuestras acciones en algún sentido: no podemos acercarnos a nuestro antojo a la realidad, hay "algo ahí" que nos limita. La naturaleza puede cooperar o no con nuestros planes. De acuerdo con el autor, referir a la "resistencia" o "cooperación" de la naturaleza es metafórico, pero constituye quizá "lo mejor que podemos hacer al tratar de capturar el sentido de la existencia de una realidad externa no arbitraria" (2012a, p. 216).

Desde esta perspectiva, respecto de las clases naturales, algo impide que clasifiquemos de cualquier modo. Si bien las clasificaciones no deben ser interpretadas en un sentido esencialista, no son arbitrarias ni meramente convencionales, porque la realidad puede hacer fracasar un sistema de clasificación y dar éxito a otro. 0 , expresado desde la perspectiva opuesta, si bien la realidad no está determinada por nuestra voluntad, sino que frustra ciertas clasificaciones y permite otras, "el mundo mismo no viene netamente dividido en cajas taxonómicas. Tenemos que inventar esas cajas nosotros mismos, y probablemente habrá cosas que no quepan bien en ningún sistema de cajas que nosotros tracemos" (Chang 2012a, p. 213). La realidad externa al sujeto existe: no determina unívocamente las clasificaciones científicas, pero las restringe en función de su éxito pragmático. 
Chang caracteriza su realismo y su pluralismo como ideológicos, en el sentido de que deben guiar nuestras acciones: el realismo científico nada tiene que ver con la adopción de determinados compromisos metafísicos; es una instancia que nos compromete con "exponernos a nosotros mismos a la realidad" y "aprender tanto como sea posible a partir de la experiencia" (Chang 2012a, p. 217). Debe abandonarse por completo la idea de que la adopción de un sistema de práctica obliga al rechazo de un sistema de práctica alternativo. Por el contrario, es conveniente cultivar simultáneamente sistemas de práctica tan inconmensurables entre sí como sea posible.

El realismo activo y el pluralismo epistémico de Chang resultan adecuados para enfrentar el problema de las clasificaciones en química. Especialmente, la noción de sistema de práctica y el abandono de una noción correspondentista de la verdad recogen la peculiaridad de la actividad científica propia de la química, basada más en el hacer, incluso el crear nuevas entidades, que en describir y predecir el comportamiento de lo que existe con independencia de nuestras acciones. Desde esta perspectiva, las clasificaciones químicas no pretenden describir el mundo tal cual es, dado que es posible tener éxito en ciencia sin conocer jamás la verdad. El ideal del realismo activo de Chang no es alcanzar descripciones verdaderas de la realidad, sino la búsqueda continua y pluralista de un conocimiento entendido en términos de praxis: para el desarrollo científico es más fértil ofrecer múltiples sistemas de práctica, incompatibles entre sí. Cuando esta posición filosófica se aplica al caso de las clasificaciones, no hay problema alguno en aceptar clasificaciones múltiples, no convergentes, que se solapen o no, incluso incompatibles. Pero, además, hay que propugnar la búsqueda de ese tipo de clasificaciones; la propia práctica científica muestra la fecundidad de tal estrategia metodológica.

Los tres problemas presentados pueden, entonces, abordarse desde la perspectiva del realismo activo de Chang. Si bien ni el número atómico ni otras propiedades microscópicas pueden considerarse las esencias que determinan la identidad de los elementos como clases naturales, ello no implica que la clasificación de los elementos químicos deba abandonarse o considerarse como meramente arbitraria. Por el contrario, resulta científicamente fructífero tomar como fecundas las diferentes formas de clasificación en sus propios contextos. En ciertos casos, el número atómico puede resultar suficiente para clasificar los elementos, pero en otros, pueden resultar más adecuadas otras clasificaciones: por ejemplo, cuando nos interesan las posibles reacciones de las especies químicas, la clasificación en términos de 
iones puede ser preferible; o cuando consideramos las propiedades macroscópicas, puede resultar más conveniente tomar como clases las variedades alotrópicas; o si nos ocupamos de los decaimientos radioactivos, la tabla de radioisótopos puede reemplazar a la tabla periódica estándar. Podemos admitir que no hay propiedades esenciales que determinen el comportamiento de los ácidos o que den cuenta de las propiedades físicas y químicas de las sustancias en todos los niveles, pero que tales clasificaciones - elementos, sustancias, ácidos y bases, por ejemplo - tampoco son arbitrarias.

En síntesis, ¿̨ómo concebir las clases en química? El pluralismo activo de Chang permite afirmar que no es necesario abandonar la idea de clases naturales, aun si, como manifiesta la propia práctica química, no es posible hallar la esencia microestructural que nos permita dar con las clasificaciones últimas y definitivas. Las clases naturales pueden mantenerse desde una perspectiva que no pretende que la ciencia ofrezca una descripción literalmente verdadera de la realidad: aquéllas no recogen, no reflejan la estructura intrínseca de la realidad, con independencia de las actividades que los sujetos realicen sobre ella. De este modo, es posible rechazar una noción correspondentista de la verdad manteniendo un compromiso realista que afirma la existencia de una realidad externa que no determina pero restringe. Podemos sostener, entonces, que "algo" en la realidad nos compele a clasificar de determinada manera en determinados contextos. Los problemas que hemos visto indican, precisamente, que no hay clases naturales transcontextuales, en un sentido esencialista y metafísico. Pero podemos afirmar que hay clases relativas a cada contexto de investigación y práctica. Si bien, desde una perspectiva realista - mínima, pero realista al fin-, estas clases están recogiendo algo de lo que está ahí, diversas clasificaciones pueden resultar correctas en distintos ámbitos. Un pluralismo epistémico basado en la praxis ofrece un marco filosófico adecuado para dar respuesta a los problemas que las clasificaciones presentan en química. Así como "el agua es $\mathrm{H}_{2} \mathrm{O}$ y también es realmente otras cosas" (Chang 2012a, p. 203), el carbono es el elemento con número atómico $\mathrm{Z}=6$ y también es realmente otras cosas.

\section{Conclusiones y perspectivas}

En el marco del problema de las clases naturales en química, y a la luz de tres dificultades específicas de la disciplina, en el presente trabajo nos hemos opuesto al realismo metafísico de aquellos que 
creen que la realidad está bien definida de una vez por todas, independientemente de la acción humana y del pensamiento humano, [...] que el propósito primordial de la ciencia es desarrollar justamente ese género de discurso que articula adecuadamente la realidad [...] y que la ciencia moderna esencialmente está logrando ese propósito. (Torretti 2007, p. 81)

Frente a esta posición, hemos defendido una postura pluralista que se aleja tanto del convencionalismo como del esencialismo respecto de las clases naturales.

Cabe aquí señalar la diferencia entre dos tipos de pluralismo que podrían haber servido para respaldar nuestra postura. El pluralismo ontológico de raíz kantiana de Lombardi y Pérez Ransanz se inspira explícitamente en la física teórica: la constitución de las ontologías científicas viene dada por los esquemas conceptuales que subyacen detrás de las teorías, expresados por los lenguajes en los que éstas se formulan. Precisamente por su vínculo con las formulaciones teóricas de la ciencia, este pluralismo puede no ser el más adecuado para pensar la química, una disciplina que no se basa en marcos teóricos amplios y fuertemente articulados: ya desde sus orígenes en la alquimia, la química se instituyó como una disciplina eminentemente práctica, con propósitos más tecnológicos que cognoscitivos. Como afirma Bernardette Bensaude-Vincent (2008), la ontología de la química surge no sólo a partir de lo cognitivo, sino también de la acción impulsada por la utilidad y la eficiencia. Por lo tanto, el realismo activo de Chang, que no descansa sobre el supuesto de la ciencia como saber proposicional, sino que se basa en la idea de sistemas de práctica, se presenta como mucho más adecuado para abordar las problemáticas filosóficas propias de la química.

Por último, cabe señalar que el presente trabajo se encuentra en la línea de una intuición que pretendemos continuar profundizando en futuros trabajos: la química se nos presenta como un campo que tiende a trastornar el tratamiento habitual de los problemas filosóficos. A diferencia de lo que ocurre en filosofía general de la ciencia, o incluso en filosofía de la física, en filosofía de la química la estrategia de definir, presentar, analizar un problema filosófico y luego buscar su aplicación en ciencia suele tropezar con las peculiaridades de la propia disciplina, que se resisten al análisis mediante los recursos filosóficos usuales. Las categorías ontológicas clásicas, como las de individuo y propiedad, ciertas caracterizaciones filosóficas establecidas, como la de clase natural, o algunas posiciones dominantes, como 
el reduccionismo microestructuralista, suelen revelarse insuficientes para pensar los problemas de la práctica química e invitan a subvertir, entonces, los abordajes tradicionales. Frente a ello, es necesario atender primero a los problemas específicos de la disciplina, para luego elaborar las herramientas filosóficas necesarias para su abordaje. En otras palabras, la química nos invita a recorrer el camino desde la disciplina hacia la filosofía y no el camino inverso, a trazar un recorrido desde los problemas científicos a los conceptos filosóficos, para luego, por supuesto, volver sobre aquéllos. Esta actitud dialógica puede resultar en un renovado crecimiento y enriquecimiento no sólo de la filosofía de la química, sino también de la filosofía general de la ciencia. ${ }^{7}$

\section{BIBLIOGRAFÍA}

Bensaude-Vincent, B., 2008, "Chemistry beyond the 'Positivism vs. Realism' Debate", en Ruthenberg y van Brakel 2008, pp. 45-53.

Bird, A. y Tobin, E., 215, "Natural kinds", en The Stanford Encyclopedia of Philosophy, ed. E.N. Zalta, edición de primavera de 2015, URL = http://plato.stanford.edu/archives/spr2015/entries/natural-kinds/

Chang, H., 2012a, Is Water $\mathrm{H}_{2} \mathrm{O}$ ? Evidence, Realism and Pluralism, Springer, Dordrecht.

— 2012b, "Acidity: The Persistence of the Everyday in the Scientific", Philosophy of Science, vol. 79, no. 5, pp. 690-700.

- 2011, "The Persistence of Epistemic Objects through Scientific Change", Erkenntnis, vol. 75, no. 3, pp. 413-429.

— , 2004, Inventing Temperature: Measurement and Scientific Progress, Oxford University Press, Nueva York.

Córdoba, M., M. Labarca, y A. Zambon, 2013, “Acerca de la unicidad de la sustancia en química", Revista Colombiana de Filosofía de la Ciencia, vol. 13, no. 26, pp. 167-180.

Córdoba, M. y O. Lombardi, 2012, “A Kantian Perspective for the Philosophy of Chemistry", en J.-P. Llored (comp.), The Philosophy of Chemistry: Practices, Methodologies, and Concepts, Cambridge Scholars Publishing, Cambridge, pp. 478-490.

Córdoba, M. y J.C. Martínez González, 2014, "Los orbitales cuánticos y la autonomía del mundo químico", Theoria, vol. 29, no. 2, pp. 261-279.

${ }^{7}$ Los autores agradecen a Olimpia Lombardi su pormenorizada lectura del presente trabajo, así como sus comentarios y sugerencias. El presente trabajo ha sido realizado gracias al apoyo del subsidio 57919 de la John Templeton Foundation y de los recursos suministrados por el Consejo Nacional de Investigaciones Científicas y Técnicas (CONICET). 
Dupré, J., 2001, “Natural Kinds", en W.H. Newton-Smith (comp.), A Companion to the Philosophy of Science, Blackwell, Oxford, pp. 311-319.

- 1993, The Disorder of Things: Metaphysical Foundations of the Disunity of Science, Harvard University Press, Cambridge, Mass.

Ellis, B., 2008, "Essentialism and Natural Kinds", en S. Psillos y M. Curd (comps.), The Routledge Companion to Philosophy of Science, Routledge, Londres, pp. 139-148.

Fortin, S., O. Lombardi y J.C. Martínez González, 2016, "Isomerism and Decoherence", Foundations of Chemistry, vol. 18, no. 3, pp. 225-240.

Frege, G., 1973 [1892], "Sobre sentido y referencia", en T.M. Simpson (comp.), Semántica filosófica: problemas y discusiones, Siglo XXI, Buenos Aires, pp. 3-27.

Hendry, R., 2006, "Elements, Compounds and Other Chemical Kinds", Philosophy of Science, vol. 73, no. 5, pp. 864-875.

Hettema, H., 2012, "Reducing Chemistry to Physics. Limits, Models, Consecuences", tesis doctoral, University of Groningen, Groninga.

Hull, D.L., 1965, "The Effect of Essentialism on Taxonomy: 2000 Years of Stasis", The British Journal for the Philosophy of Science, vol. 15, no. 60 , pp. 314-326.

Kripke, S., 1980, Naming and Necessity, Basil Blackwell, Oxford.

—_, 1972, "Naming and Necessity", en G. Harman y D. Davidson (comps.), Semantics of Natural Language, Reidel, Dordrecht, pp. 253355.

Leach, M., 2013, "Concerning Electronegativity as a Basic Elemental Property and Why the Periodic Table Is Usually Represented in Its Medium Form", Foundations of Chemistry, vol. 15, no. 1, pp. 13-29.

Lewis, G., 1923, Valence and the Structure of Atoms and Molecules, Dover, Nueva York.

Locke, J., 1980 [1690], Ensayo sobre el entendimiento humano, Editora Nacional, Madrid.

Lombardi, O. y Castagnino, M., 2010, "Matters Are Not So Clear on the Physical Side", Foundations of Chemistry, vol. 12, no. 2, pp. 159-166.

Lombardi, O. y M. Labarca, 2005, "The Ontological Autonomy of the Chemical World", Foundations of Chemistry, vol. 7, no. 2, pp. 125-148.

Lombardi, O. y A.R. Pérez Ransanz, 2012, Los múltiples mundos de la ciencia. Un realismo pluralista y su aplicación a la filosofía de la física, Siglo XXI, México.

—_ 2008, "Lenguaje, ontología y relaciones interteóricas: en favor de un genuino pluralismo ontológico", Revista Arbor. Ciencia, Pensamiento y Cultura, vol. 187, no. 747, pp. 43-52.

Mach, E., 1984 [1886], The Analysis of Sensations, Open Court, La Salle.

Needham, P., 2008, "A Critique of the Kripke/Putnam Conception of Water", en van Brakel y Ruthenberg 2008, pp. 93-105.

—, 2000, “What is Water?", Analysis, vol. 60, no. 265, pp. 13-21. 
Paneth, F.A., 1962a, "The Epistemological Status of the Chemical Concept of Element" (Parte I), British Journal for the Philosophy of Science, vol. 13, no. 49, pp. 1-14 [reimpreso en Foundations of Chemistry, vol. 5, no. 2, 2003, pp. 113-145].

__ 1962b, "The Epistemological Status of the Chemical Concept of Element" (Parte II), British Journal for the Philosophy of Science, vol. 13, no. 50, pp. 144-160 [reimpreso en Foundations of Chemistry, vol. 5, no. 2, 2003, pp. 113-145].

Psillos, S., 1999, Scientific Realism: How Science Tracks Truth, Routledge, Nueva York/Londres.

Putnam, H., 1975, Mind, Language and Reality, Philosophical Papers II, Cambridge University Press, Cambridge.

- 1973, "Meaning and Reference", Journal of Philosophy, vol. 70, no. 19, pp. 699-711.

Russell, B., 1905, “On Denoting”, Mind, vol. 14, no. 56, pp. 479-493.

Ruthenberg, K. y J. van Brakel (comps.), 2008, Stuff: The Nature of Chemical Substances, Königshausen u. Neumann, Würzburg.

Scerri, E., 2005, "Some Aspects of the Metaphysics of Chemistry and the Nature of the Elements", Hyle - International Journal for Philosophy of Chemistry, vol. 11, no. 2, pp. 127-145.

- 1997, "Has the Periodic Table Been Successfully Axiomatized?", Erkenntnis, vol. 47, no. 2, pp. 229-243.

Schwarz, W.H.E., 2007, "Recommended Questions on the Roads towards a Scientific Explanation of the Periodic System of the Chemical Elements with the Help of the Concepts of Quantum Physics", Foundations of Chemistry, vol. 9, no. 2, pp. 139-188.

Searle, J., 1958, "Proper Names", Mind, vol. 67, no. 266, pp. 166-173.

Strawson, P.F., 1959, Individuals: An Essay in Descriptive Metaphysics, Methuen, Londres.

Sutcliffe, B. y R.G. Wolley, 2012, "Atoms and Molecules in Classical Chemistry and Quantum Mechanics", en R.F. Hendry y A. Woody (comps.), Handbook of Philosophy of Science, vol. 6, Philosophy of Chemistry, Elsevier, Oxford, pp. 387-426.

Torretti, R., 2007, "El realismo científico y la ciencia como es", en Escritos filosóficos 1986-2006, Universidad Diego Portales, Santiago de Chile, pp. 75-98.

Van Brakel, J., 2012, "Substances: The Ontology of Chemistry", en A. Woody, R. Hendry y P. Needham (comps.), Philosophy of Chemistry, Elsevier, Amsterdam, pp. 191-229.

- 2000, "The Nature of Chemical Substances", en N. Bhushan y S. Rosenfeld (comps.), Of Minds and Molecules. New Philosophical Perspectives on Chemistry, Oxford University Press, New York, pp. 162184.

Van der Vet, 1979, "The Debate between F.A. Paneth, G. von Hevesy, and K. Fajans on the Concept of Chemical Identity", Janus, vol. 66, pp. 285-303. 
Weisberg, M., 2005, "Water is not $\mathrm{H}_{2} \mathrm{O}$ ", en D. Baird, E. Scerri y L. McIntyre (comps.), Philosophy of Chemistry: Synthesis of a New Discipline, Springer, Dordrecht, pp. 337-345.

Recibido el 20 de abril de 2016; revisado el 4 de octubre de 2016; aceptado el 2 de diciembre de 2016. 